\title{
New Aspects of Physics of Magnetic Resonance and Its Applications
}

\author{
Vladimir I. Chizhik ${ }^{1}$ Murat S. Tagirov ${ }^{2}$
}

(C) Springer-Verlag Wien 2017

Magnetic resonance has widely been used to study condensed media since the observation of EPR (1944) and NMR (1946) in these objects. The goal of this special issue is to set off some perspective directions in the area of the magnetic resonance (MR) and its applications. Although the papers collected in this issue cannot cover all aspects of modern trends, they may give a flavor on the current state of the art of interesting researches. Let us make a brief review of the content of this issue.

First of all, we would like to underline articles, in which fundamentally new approaches in the methods of the MR and its applications are presented:

Yu. M. Bunkov, A. V. Klochkov, T. R. Safin, K. R. Safiullin, and M. S. Tagirov investigate the Bose-Einstein condensation (BEC) of magnons in nuclear magnetic resonance experiments with several solid-state antiferromagnets; it has been found that the magnon BEC suppresses the formation of a spin echo signal that confirms the formation of the magnon BEC state in considered systems.

M. S. Kuznetsova presents the original results of the investigation of nuclear spin dynamics in nanostructures with negatively charged InGaAs/GaAs quantum dots characterized by the strong quadrupole splitting of nuclear spin sublevels; the main methods of the investigation are experimental measurements and theoretical analysis of the photoluminescence polarization as the function of the transverse magnetic field (effect Hanle); experimental data are analyzed using an original approach based on the separate consideration of behavior of the longitudinal and

Vladimir I. Chizhik

v.chizhik@spbu.ru

Murat S. Tagirov

Murat.Tagirov@kpfu.ru

1 Saint Petersburg State University, Saint Petersburg, Russia

2 Kazan Federal University, Kazan, Russia 
transverse components of nuclear polarization; a new effect of resonant optical pumping of nuclear spin polarization in an ensemble of singly charged (In, Ga)As/ GaAs quantum dots subjected to a transverse magnetic field is observed.

The problems of molecular dynamics and spin relaxation are considered in the following articles:

M. Dolgushev, S. Schnell, and D. A. Markelov study the role of hydrodynamic interactions for the relaxation of bonds' orientations in dendrimers; they show that the correlations between bond orientations play a major role for the mobility of segments that is manifested in the NMR relaxation functions.

V. Klimavicius, L. Dagys, V. Chizhik, and V. Balevicius carried out the high data point density measurements of the ${ }^{1} \mathrm{H}^{11} \mathrm{~B}$ cross-polarization $(\mathrm{CP})$ kinetics upon MAS for the ionic liquid [bmim] $\left[\mathrm{BF}_{4}\right]$ confined in mesoporous SBA-15 and MCM41; the complex shaped ${ }^{11} \mathrm{~B}$ CP MAS signals were observed in both silica that points towards the possibility of the bimodal distribution of $[\mathrm{bmim}]\left[\mathrm{BF}_{4}\right]$ in the confinements studied; the convergence of classical and non-classical spin coupling models is deduced processing CP kinetic curves.

A few articles are devoted to the magnetic resonance imaging (MRI) and related questions, in particular, to MRI in weak magnetic fields (MRI in high magnetic fields is contraindicated for certain groups of patients):

V. Chizhik, V. Frolov, P. Kupriyanov, and K. Tyutyukin consider the problem of reduction of the effect of concomitant gradients in low magnetic field MRI via optimization of gradient magnetic system; the manifestation of concomitant magnetic fields in a concrete gradient system, namely in the system of two coaxial gradient coils, is thoroughly analyzed and it has been shown that in the case of low fields (like the Earth field), the optimal interspace is not the Maxwell condition.

S. V. Ievleva, N. V. Luzhetckaia, K. V. Tyutyukin, and V. V. Frolov present the results of the first experiments with diffusion weighted imaging in such low magnetic field as $7 \mathrm{mT}$.

A. A. Bogdan, G. V. Kataeva, J. G. Khomenko, A. G. Ilves, and L. N. Prakhova analyze the quality of the signals received from metabolites in ${ }^{1} \mathrm{H}$ magnetic resonance spectroscopy (MRS); they compare MRS (Achieva 3T 2D PRESS 1HMRS $(\mathrm{TE}=53$ and $144 \mathrm{~ms}, \mathrm{TR}=2000 \mathrm{~ms}$ ) data acquired in supraventricular white matter and medial cortex at two various TE (53 and $144 \mathrm{~ms}$ ) for patients with the multiple sclerosis (25 patients with the confirmed diagnosis of relapsingremitting multiple sclerosis and 20 patients with the diagnosis of secondary progressive multiple sclerosis) and control group (21 healthy volunteers, comparable on age), to evaluate advantages and disadvantages of different echo time in the clinical practice.

Yu. V. Bogachev, A. V. Nikitina, A. A. Kostina, V. A. Sabitova, V. V. Pankov, T. G. Shutava, E. G. Petrova, D. A. Kotsikau, V. O. Natarov, and K. S. Livanovich describe the results of proton NMR relaxation measurements in aqueous solutions of MgxZnyFe3-x-yO4 magnetite-based composite magnetic nanoparticles (MNPs); it is shown that carbonate-synthesized MNPs possess higher relaxation efficiency 
(relaxivity) as compared with base-precipitated complex oxides that can be useful in MRI contrasting.

A very interesting development of MR applications is presented in the article by E. Alakshin, R. Gazizulin, A. Klochkov, E. Kondratyeva, A. Laskin, and M. Tagirov. They discuss the advantages of proposed ${ }^{3} \mathrm{He}$ porometry method; geological samples of calcium carbonates $\left(\mathrm{CaCO}_{3}\right)$ were investigated by ${ }^{3} \mathrm{He}$ NMR, nitrogen porosimetry, X-ray diffraction, and electron microscopy.

At the end of the issue, there are two articles in which specific problems are solved:

D. S. Mazing, A. V. Nikiforova, A. S. Osinin, V. A. Moshnikov, Yu. V. Bogachev, and S. M. Sukharzhevskii entitled "Electron Paramagnetic Resonance Investigations of ZnSe: Mn Nanocrystals".

B. Wu, S. A. Altobelli, and E. Fukushima entitled "Single-sequence multi-slice NMR in strong gradient magnetic fields".

We hope that the publications presented in this issue will stimulate further progress in the magnetic resonance and its applications. We are grateful to all authors who contributed to this special issue. 